\title{
ON THE ORDER OF THE AUTOMORPHISM GROUP OF FOLIATIONS
}

\author{
MAURÍCIO CORRÊA J.R AND THIAGO FASSARELLA
}

\begin{abstract}
Let $\mathcal{F}$ be a holomorphic foliation with ample canonical bundle on a smooth projective surface $X$. We obtain an upper bound on the order of its automorphism group which depends only on $K_{\mathcal{F}}^{2}$ and $K_{\mathcal{F}} K_{X}$, provided this group is finite. Here, $K_{\mathcal{F}}$ and $K_{X}$ are the canonical bundles of $\mathcal{F}$ and $X$, respectively.
\end{abstract}

\section{INTRODUCTION}

A finiteness theorem for the automorphism group of a smooth curve of genus at least 2 goes back to the 19th century with H. A. Schwarz, F. Klein and H. Poincaré. The order of this group was studied by A. Hurwitz who obtained in [12] the following well-known bound

$$
|\operatorname{Aut}(C)| \leq 42 \operatorname{deg}\left(K_{C}\right),
$$

where $K_{C}$ is the canonical bundle of the curve $C$. In arbitrary dimension, the finiteness of the automorphism group, or more generally, of the group of birational self-maps was proved by A. Andreotti in [1] for varieties of general type, and a bound in the above sense, was obtained in the 2 -dimensional case. He showed that there exists a universal function $f$ so that

$$
|\operatorname{Aut}(X)| \leq f\left(c_{2}\right),
$$

where $c_{2}$ is the second Chern class of the tangent bundle of the smooth surface $X$. The function $f$ given by Andreotti is of exponential type, and since then this estimate has been improved in [11, 6, 20, 21 and extended for higher dimensions by many others in [10, 5, 18, 22, 19, 23, 9], placed in chronological order.

In the present paper, we are interested in the subgroup of the automorphism group, or even of the group of birational self-maps, that preserves a holomorphic foliation $\mathcal{F}$ on a smooth projective surface $X$. One of the first progress in this direction has been made by J. V. Pereira and P. F. Sanchez in [16]. They showed that the group $\operatorname{Bir}(\mathcal{F})$ of birational self-maps preserving a foliation of general type on a projective surface is finite. Therefore, we can naturally to raise the question if there exists an upper bound for the order of $\operatorname{Bir}(\mathcal{F})$ in the sense of the previous one.

One purpose of this work is to answer this question for a particular class of general type foliations, namely, those whose the canonical bundle $K_{\mathcal{G}}$ of a reduced model $\mathcal{G}$ of $\mathcal{F}$ is ample, see Corollary 4.4. This follows as consequence of Theorem 4.2, our main result, where we obtain an explicit upper bound on the order of $\operatorname{Aut}(\mathcal{F})$ in which depends only on intersections of the canonical bundles of $\mathcal{F}$ and $X$. More precisely, this bound is a function of $K_{\mathcal{F}}^{2}$ and $K_{\mathcal{F}} K_{X}$. In our theorem, a finiteness hypothesis on $\operatorname{Aut}(\mathcal{F})$ is necessary. We point out that foliations on projective surfaces having infinite automorphism group are very rare and they have been classified, up to birational maps, in 4 by S. Cantat and C. Favre. As a consequence of this classification, in Proposition 2.2 we classify the foliations on projective surfaces having infinite automorphism group and ample canonical bundle.

This paper is organized as follows. In Section 2 we recall some basic concepts on codimension one foliations as well their canonical bundles. In Section 3, we obtain a polynomial upper bound on the order of the automorphism group of a codimension one $k$-web of degree $d$ on $\mathbb{P}^{N}$ in terms of $k, d$ and $N$, and introduce their dual pairs. Finally, in Section 4 , we prove our main results.

ACKnowledgements. We are greatly indebted to Charles Favre who have shown us the way to prove Proposition 2.2. We also wish to thank Javier Ribón, Maycol Falla and Nivaldo Medeiros for many stimulating conversations on this subject. 


\section{Codimension One Foliations}

2.1. Basic Definitions. Let $X$ be a smooth complex variety of dimension $n>1$. A codimension one foliation $\mathcal{F}$ on $X$, is given by an open covering $\mathcal{U}=\left\{U_{i}\right\}$ of $X$ and 1-forms $\omega_{i} \in \Omega_{X}^{1}\left(U_{i}\right)$ subject to the conditions:

(1) For each non-empty intersection $U_{i} \cap U_{j}$ there exists a function $g_{i j} \in \mathcal{O}_{U_{i} \cap U_{j}}^{*}$ such that $\omega_{i}=g_{i j} \omega_{j}$

(2) For every $i$ the zero set of $\omega_{i}$ has codimension at least two;

(3) For every $i, \omega_{i} \wedge d \omega_{i}=0$.

The 1-forms $\left\{\omega_{i}\right\}$ patch together to form a global section

$$
\omega=\left\{\omega_{i}\right\} \in \mathrm{H}^{0}\left(X, \Omega_{X}^{1} \otimes \mathcal{L}\right),
$$

where $\mathcal{L}$ is the line bundle over $X$ determined by the cocycle $\left\{g_{i j}\right\}$. The singular set of $\mathcal{F}$, denoted by $\operatorname{Sing}(\mathcal{F})$, is the zero set of the twisted 1 -form $\omega$.

Let $T X$ be the tangent sheaf of $X$. The tangent sheaf of $\mathcal{F}$, induced by a twisted 1 -form $\omega \in \mathrm{H}^{0}\left(X, \Omega_{X}^{1} \otimes \mathcal{L}\right)$, is defined on each open set $U \subset X$ by

$$
T_{\mathcal{F}}(U)=\left\{v \in T X(U) ; i_{v} \omega=0\right\} .
$$

The dual of $T_{\mathcal{F}}$ is the cotangent sheaf of $\mathcal{F}$ and will be denoted by $T_{\mathcal{F}}^{*}$. The determinant of $T_{\mathcal{F}}^{*}$, i.e. $\left(\wedge^{n-1} T_{\mathcal{F}}^{*}\right)^{* *}$, is called the canonical bundle of $\mathcal{F}$ and is denoted by $K_{\mathcal{F}}$. If $n=2$, we can use vector fields instead 1 -forms. The foliation can be given by $v_{i} \in T X\left(U_{i}\right)$ with codimension two zero set and satisfying $v_{i}=f_{i j} v_{j}$, where $f_{i j} \in \mathcal{O}_{U_{i} \cap U_{j}}^{*}$. In this case, $K_{\mathcal{F}}$ is the line bundle determined by the cocycle $\left\{f_{i j}\right\}$. For example, if $\mathcal{F}$ is given by a (non-trivial) global vector field $v \in \mathrm{H}^{0}(X, T X)$ and $D$ denotes the zero divisor of $v$ (possibly empty if $v$ has codimension two zero set) then $K_{\mathcal{F}}=\mathcal{O}_{X}(-D)$.

The condition (3) together with Frobenius Theorem imply that for every point in the complement of $\operatorname{Sing}(\mathcal{F})$, there exists a unique germ of smooth codimension one hypersurface $V$ invariant by $\mathcal{F}$, i.e., satisfying $i^{*}(\omega)=0$ where $i: V \longrightarrow X$ is the inclusion. Analytic continuation of these subvarieties describes the leaves of $\mathcal{F}$. As usual, we will make abuse of notation by writing $\left(T_{\mathcal{F}}\right)_{x}$ for the tangent space to the leaf passing through $x$ or for the stalk of the sheaf $T_{\mathcal{F}}$ at $x$.

We say that $\varphi: X \longrightarrow X$ preserves $\mathcal{F}$ if it sends leaves on leaves, that is if $\varphi^{*} \omega$ defines the same foliation $\mathcal{F}$. Through this work we want to analyze the order of the groups $\operatorname{Aut}(\mathcal{F})$ and $\operatorname{Bir}(\mathcal{F})$, which are respectively defined as the maximal subgroup of automorphisms $\operatorname{Aut}(X)$ and of birational self-maps $\operatorname{Bir}(X)$ that preserve $\mathcal{F}$. It has been proved by J. V. Pereira and P. Sanchez in [16] the following finiteness theorem for general type foliations on surfaces.

Theorem 2.1. If $\mathcal{F}$ is a foliation of general type on a smooth projective surface then $\operatorname{Bir}(\mathcal{F})$ is finite.

A foliation on a surface $X$ is said of general type if the Kodaira dimension of $\mathcal{F}, \operatorname{Kod}(\mathcal{F})$, is equal to 2. The concept of Kodaira dimension for holomorphic foliations has been introduced independently by L. G. Mendes and M. McQuillan. For more information on the subject see [2]. For convenience to the reader we will recall it in the next few lines.

An isolated singularity $x$ of $\mathcal{F}$ is called reduced if the eigenvalues of the linear part $D v(x)$ are not both zero and their quotient, when defined, is not a positive rational number. A foliation is called reduced if all the singularities are reduced. A remarkable theorem by A. Seidenberg (see [17]) says that there exists a sequence of blowing-ups $\pi: \tilde{X} \longrightarrow X$ over singularities of $\mathcal{F}$ such that the induced foliation $\pi^{*}(\mathcal{F})$ in $\tilde{X}$ has only reduced singularities. Any reduced foliation birationaly equivalent to $\mathcal{F}$ is called reduced model of $\mathcal{F}$.

We could define the Kodaira dimension of $\mathcal{F}$ as the Kodaira dimension of the line bundle $K_{\mathcal{F}}$, for short $\operatorname{Kod}\left(K_{\mathcal{F}}\right)$. But this is not a birational invariant for $\mathcal{F}$. For example, the radial foliation (lines passing through a point) on $\mathbb{P}^{2}$ has canonical bundle $K_{\mathcal{F}}=\mathcal{O}_{\mathbb{P}^{2}}(-1)$ but a suitable birational transformation $\mathbb{P}^{2} \rightarrow \mathbb{P}^{2}$ defined by polynomials of high degree transforms it into a foliation $\mathcal{G}$ with canonical bundle $K_{\mathcal{G}}=\mathcal{O}_{\mathbb{P}^{2}}(d-1)$. Fortunately, $\operatorname{Kod}\left(K_{\mathcal{F}}\right)$ is a birational invariant 
for foliations having only reduced singularities. So if $\mathcal{F}$ is a foliation on the complex surface $X$, and $\mathcal{G}$ is any reduced model of $\mathcal{F}$, the Kodaira dimension of $\mathcal{F}$ is defined as $\operatorname{Kod}\left(K_{\mathcal{G}}\right)$.

2.2. Foliations with $K_{\mathcal{F}}$ ample on surfaces. One of the main propose of this paper is to give a universal bound for the order of $\operatorname{Aut}(\mathcal{F})$ by supposing this group is finite and also that $K_{\mathcal{F}}$ is ample. Foliations having infinite automorphism group were classified up to birational maps by S. Cantat and C. Favre in [4 in the 2-dimensional case. In what follows we will make use of their ideas to classify the foliations on projective surfaces having infinite automorphism group and ample canonical bundle.

Let $X$ be a projective surface and $f: X \longrightarrow X$ an automorphism. Fix an ample line bundle $\mathcal{L}$ on $X$. The degree of $f$ with respect to $\mathcal{L}$ is defined as

$$
\operatorname{deg}_{\mathcal{L}}(f)=f^{*} \mathcal{L} \cdot \mathcal{L} .
$$

It is known that the asymptotic behavior of the sequence $\operatorname{deg}_{\mathcal{L}}\left(f^{n}\right)$ is independent of the chosen line bundle $\mathcal{L}$. See for example the proof of Proposition 3.1 in [3. Furthermore, this sequence either can be bounded or can have growth of the following types: linear, quadratic or exponential. As we will see below, the case in which this sequence is bounded is particularly interesting for us. Under this hypothesis the automorphism $f$ is the time-1 map associated to a flow of a global vector field. For detail see [4, p. 7] and references therein.

Now let $\mathcal{F}$ be a foliation on $X$ with $K_{\mathcal{F}}$ ample and infinite group $\operatorname{Aut}(\mathcal{F})$. By [4 Theorem 1.1] there exists an element $f \in \operatorname{Aut}(\mathcal{F})$ with infinite order. Since $K_{\mathcal{F}}$ is ample and $f^{*}\left(K_{\mathcal{F}}\right)=K_{\mathcal{F}}$ then the sequence of degrees $\operatorname{deg}_{K_{\mathcal{F}}}\left(f^{n}\right)$ is bounded. Hence $f$ is the time-1 flow of a nontrivial global vector field $v$.

This implies that $\mathcal{F}$ is preserved by a flow of a global vector field $v \in \mathrm{H}^{0}(X, T X)$. Indeed, let $\operatorname{Aut}_{0}(X)$ be the connected component of the identity of the complex Lie group $\operatorname{Aut}(X)$ and $\operatorname{Aut}_{0}(\mathcal{F})=\operatorname{Aut}_{0}(X) \cap \operatorname{Aut}(\mathcal{F})$ be the closed Lie subgroup of $\operatorname{Aut}_{0}(X)$. If $\varphi_{t}$ is the time- $t$ flow of $v$, then $\varphi_{n}=f^{n} \in \operatorname{Aut}_{0}(\mathcal{F})$ for all $n \in \mathbb{N}$. This is enough to ensure that the Lie algebra of $\operatorname{Aut}_{0}(\mathcal{F})$ is nontrivial.

Since $\mathcal{F}$ is preserved by a flow, it follows from [4, Proposition 3.8] that $\mathcal{F}$ is one of the following types: turbulent, Riccati, rational fibration, elliptic fibration, linear foliation on a torus or up to a birational map, $\mathcal{F}$ is preserved by the flow of a vector field on $\mathbb{P}^{1} \times \mathbb{P}^{1}$. All these cases, unless the last one, cannot happen under the hypothesis of $K_{\mathcal{F}}$ to be ample. In fact, if $\mathcal{F}$ is turbulent or Riccati and $F$ is a generic fiber of the elliptic or rational fibration then $K_{\mathcal{F}} F=0$ (see [2, p. 23]). If $\mathcal{F}$ is a rational fibration then the restriction of $K_{\mathcal{F}}$ to a generic leaf $L \cong \mathbb{P}^{1}$ is $\mathcal{O}_{\mathbb{P}^{1}}(-2)$. Finally, if $\mathcal{F}$ is a linear foliation on a torus then $K_{\mathcal{F}}=\mathcal{O}_{X}$.

Suppose $\mathcal{F}$ is birational to a foliation preserved by the flow of a vector field on $\mathbb{P}^{1} \times \mathbb{P}^{1}$. The birational map from $X$ to $\mathbb{P}^{1} \times \mathbb{P}^{1}$ is obtained in the following way. When $X$ is rational we can contract $(-1)$-curves to arrive in a minimal surface, which must be either $\mathbb{P}^{2}$ or a Hirzebruch surface $\Sigma_{n}, n \geq 0$. We note that, at this point, $K_{\mathcal{F}}$ is still ample. Blowing-up a singular point of $v$ we can replace $\mathbb{P}^{2}$ by $\Sigma_{1}$. To arrive in $\mathbb{P}^{1} \times \mathbb{P}^{1}$ we have to make flipping of fibers that contains singularities of $v$ (see [2, p. 87]). The resulting foliation, still denoted by $\mathcal{F}$, on $\mathbb{P}^{1} \times \mathbb{P}^{1}$ is invariant by the flow of a vector field $v_{1} \oplus v_{2}$. In addition, the argument of S. Cantat and C. Favre in the end of the proof of Proposition 3.8 in 4 shows that $\mathcal{F}$ is given by a global vector field if $v$ is not tangent to a foliation by rational curves. We summarize the above discussion in the following proposition.

Proposition 2.2. If $\mathcal{F}$ is a foliation on a smooth projective surface $X$ with $K_{\mathcal{F}}$ ample and $\operatorname{Aut}(\mathcal{F})$ infinite, then up to a birational map, $\mathcal{F}$ is preserved by the flow of a vector field $v=v_{1} \oplus v_{2}$ on $\mathbb{P}^{1} \times \mathbb{P}^{1}$. Moreover, if $v$ is not tangent to a foliation by rational curves then $\mathcal{F}$ is given by a global vector field on $\mathbb{P}^{1} \times \mathbb{P}^{1}$.

Example 2.3. Let us give an example of a foliation on $\mathbb{P}^{2}$ with $K_{\mathcal{F}}$ ample and $\operatorname{Aut}(\mathcal{F})$ infinite. Consider the foliation $\mathcal{F}$ of degree two on $\mathbb{P}^{2}$ given in homogeneous coordinates by the 1 -form

$$
\omega=-b y z^{2} d x+\left(b x z^{2}+a z y^{2}\right) d y-a y^{3} d z
$$


where $a, b \in \mathbb{C}^{*}$. This foliation has ample canonical bundle $K_{\mathcal{F}}=\mathcal{O}_{\mathbb{P}^{2}}(1)$ and is preserved by the

flow of the global vector field $v=y \frac{\partial}{\partial x}$. In fact, the flow preserves the 1-forma $\omega$ since $L_{v} \omega=0$ where $L_{v}$ is the Lie derivative. Blowing-up two singular points of $\mathcal{F}$, contained in $(y=0)$, and contracting a (-1)-curve we arrive in a foliation given by the 1-form $b d y-a d x$ in an affine chart of $\mathbb{P}^{1} \times \mathbb{P}^{1}$ which is preserved by the flow of $\frac{\partial}{\partial x}$. We leave the details to the reader.

\section{Codimension one Webs}

3.1. Basic Definitions. Let $X$ be a smooth complex variety of dimension $n>1$. A codimension one $k$-web $\mathcal{W}$ on $X$ is given by an open covering $\mathcal{U}=\left\{U_{i}\right\}$ of $X$ and $k$-symmetric 1 -forms $\omega_{i} \in \operatorname{Sym}^{k} \Omega_{X}^{1}\left(U_{i}\right)$ subject to the conditions:

(1) For each non-empty intersection $U_{i} \cap U_{j}$ there exists a non-vanishing function $g_{i j} \in \mathcal{O}_{U_{i} \cap U_{j}}$ such that $\omega_{i}=g_{i j} \omega_{j}$;

(2) For every $i$ the zero set of $\omega_{i}$ has codimension at least two;

(3) For every $i$ and a generic $x \in U_{i}$, the germ of $\omega_{i}$ at $x$ seen as homogeneous polynomial of degree $k$ in the ring $\mathcal{O}_{x}\left[d x_{1}, \ldots, d x_{n}\right]$ is square-free;

(4) For every $i$ and a generic $x \in U_{i}$, the germ of $\omega_{i}$ at $x$ is a product of $k$ germs of 1 -forms $\left(\omega_{i}\right)_{x}=\beta_{1} \cdots \beta_{k}$ satisfying the integrability condition $\beta_{i} \wedge d \beta_{i}=0$.

The $k$-symmetric 1 -forms $\left\{\omega_{i}\right\}$ patch together to form a global section

$$
\omega=\left\{\omega_{i}\right\} \in \mathrm{H}^{0}\left(X, \operatorname{Sym}^{k} \Omega_{X}^{1} \otimes \mathcal{L}\right),
$$

where $\mathcal{L}$ is the line bundle over $X$ determined by the cocycle $\left\{g_{i j}\right\}$. Two global sections in $\mathrm{H}^{0}\left(X, \operatorname{Sym}^{k} \Omega_{X}^{1} \otimes \mathcal{L}\right)$ determine the same web if and only if they differ by the multiplication by an element of $\mathrm{H}^{0}\left(X, \mathcal{O}_{X}^{*}\right)$. The singular set of $\mathcal{W}$, denoted by $\operatorname{Sing}(\mathcal{W})$, is the zero set of $\omega$.

We say that $x \in X$ is a smooth point of $\mathcal{W}, x \in \mathcal{W}_{s m}$, if $x \notin \operatorname{Sing}(\mathcal{W})$ and the germ of $\omega$ at $x$ satisfies the conditions described in (3) and (4) above. For any smooth point $x$ of $\mathcal{W}$ we have $k$ distinct (not necessarily in general position) linearly embedded subspaces of dimension $n-1$ passing through $x$. Each one of these subspaces will be called $(n-1)$-plane tangent to $\mathcal{W}$ at $x$ and denoted by $T_{x}^{1} \mathcal{W}, \ldots, T_{x}^{k} \mathcal{W}$. Furthermore, these conditions ensure that in a neighborhood of $x$ there are $k$ distinct codimension one foliations $\mathcal{F}^{1}, \ldots, \mathcal{F}^{k}$ satisfaing $T \mathcal{F}_{x}^{j}=T_{x}^{j} \mathcal{W}$.

In the case that $X=\mathbb{P}^{N}$ the degree of $\mathcal{W}$, denoted by $\operatorname{deg}(\mathcal{W})$, is geometrically defined as the degree of the tangency locus between $\mathcal{W}$ and a generic $\mathbb{P}^{1}$ linearly embedded in $\mathbb{P}^{N}$. If $i: \mathbb{P}^{1} \hookrightarrow \mathbb{P}^{N}$ is the inclusion, then $\operatorname{deg}(\mathcal{W})$ is the degree of the zero divisor of the twisted $k$-symmetric 1 -form $i^{*} \omega \in \mathrm{H}^{0}\left(\mathbb{P}^{1},\left.\operatorname{Sym}^{k} \Omega_{\mathbb{P}^{1}}^{1} \otimes \mathcal{L}\right|_{\mathbb{P}^{1}}\right)$. Since $\Omega_{\mathbb{P}^{1}}^{1}=\mathcal{O}_{\mathbb{P}^{1}}(-2)$ one obtains $\mathcal{L}=\mathcal{O}_{\mathbb{P}^{n}}(d+2 k)$ where $d=\operatorname{deg}(\mathcal{W})$. From the Euler sequence 1 we can deduce the following

$$
0 \longrightarrow \operatorname{Sym}^{k-1}\left(\mathcal{O}_{\mathbb{P}^{N}}(1)^{\oplus(N+1)}\right) \otimes \mathcal{O}_{\mathbb{P}^{N}} \longrightarrow \operatorname{Sym}^{k}\left(\mathcal{O}_{\mathbb{P}^{N}}(1)^{\oplus(N+1)}\right) \longrightarrow \operatorname{Sym}^{k} T \mathbb{P}^{N} \longrightarrow 0 .
$$

Taking the dual sequence and tensorizing by $\mathcal{O}_{\mathbb{P}^{N}}(d+2 k)$ we can see that a $k$-web given by

$$
\omega \in \mathrm{H}^{0}\left(\mathbb{P}^{N}, \operatorname{Sym}^{k} \Omega_{\mathbb{P}^{N}}^{1} \otimes \mathcal{O}_{\mathbb{P}^{N}}(d+2 k)\right)
$$

can be represented in homogeneous coordinates by a $k$-symmetric form, still denoted by $\omega$

$$
\omega(x)=\sum_{|I|=k} A_{I}(x) d x_{0}^{i_{0}} \cdots d x_{N}^{i_{N}},
$$

whose the coefficients $A_{I}$ are homogeneous polynomials of degree $d+k$ in $x_{0}, \ldots, x_{N}$. Two $k$ symmetric forms in homogeneous coordinates define the same $k$-web if they differ by a constant.

We finish this section remarking that a $k$-web on a possible singular projective variety $X$ is a $k$-web on its smooth locus which extends to a global web on any of its desingularizations. We also observe that a 1-web is a codimension one foliation, as defined in Section 2

\footnotetext{
${ }^{1}$ For properties of symmetric powers see [7 Propositions A2.2 and A2.7].
} 
3.2. Automorphism Group of Projective Webs. Let $\mathcal{W}$ be a codimension one $k$-web on the complex projective space $\mathbb{P}^{N}$ defined by the twisted $k$-symmetric 1 -form $\omega$. We say that $T \in \operatorname{Aut}\left(\mathbb{P}^{N}\right)$ preserves $\mathcal{W}$ if the pullback $T^{*} \omega$ determines the same $k$-web $\mathcal{W}$. As remarked in the last section, $\omega$ and $T^{*} \omega$ must differ by the multiplication by an element of $\mathrm{H}^{0}\left(\mathbb{P}^{N}, \mathcal{O}_{\mathbb{P} N}^{*}\right)$, that is, by a nonvanishing constant. The automorphism group of $\mathcal{W}$, denoted by $\operatorname{Aut}(\mathcal{W})$, is the maximal subgroup of $\operatorname{Aut}\left(\mathbb{P}^{N}\right)$ that preserves $\mathcal{W}$.

Proposition 3.1. Let $\mathcal{W}$ be a $k$-web on $\mathbb{P}^{N}, N \geq 2$, of degree d and finite automorphism group. Then

$$
|\operatorname{Aut}(\mathcal{W})| \leq(d+2 k)^{(N+1)^{2}-1}
$$

Proof. We first consider the $k$-symmetric form defining $\mathcal{W}$ in homogeneous coordinates

$$
\omega(x)=\sum_{|I|=k} A_{I}(x) d x_{0}^{i_{0}} \cdots d x_{N}^{i_{N}}
$$

where $A_{I}\left(x_{0}, \ldots, x_{N}\right)$ is a homogeneous polynomial of degree $d+k$ for all $I=\left(i_{0}, \ldots, i_{N}\right)$.

Writing an element of $\operatorname{Aut}(\mathcal{W})$ as a $(N+1) \times(N+1)$ matrix we may think of its coordinates as homogeneous coordinates of a point $T=\left(\ldots: a_{i j}: \ldots\right) \in \mathbb{P}^{r}$ where $r=(N+1)^{2}-1$.

Since $\omega$ and $T^{*} \omega$ differ by the multiplication by a nonvanishing constant, for fixed $x=$ $\left(x_{0}, \ldots, x_{N}\right)$ the entries of $T$ must satisfy the equation $\omega(x) \wedge\left(T^{*} \omega\right)(x)=0$. Now we can rewrite the pullback of $\omega$ by $T$,

$$
T^{*} \omega(x)=\sum_{|I|=k} A_{I}\left(\ldots, \sum a_{i j} x_{j}, \ldots\right)\left(\sum a_{0 j} d x_{j}\right)^{i_{0}} \ldots\left(\sum a_{N j} d x_{j}\right)^{i_{N}}
$$

in terms of the basis $\left\{d x_{0}^{i_{0}} \cdots d x_{N}^{i_{N}} ;|I|=k\right\}$ to obtain

$$
T^{*} \omega(x)=\sum_{|I|=k} B_{I}^{x}\left(\ldots, a_{i j}, \ldots\right) d x_{0}^{i_{0}} \cdots d x_{N}^{i_{N}}
$$

where $B_{I}^{x}\left(\ldots, a_{i j}, \ldots\right)$ is a homogeneous polynomial of degree $d+2 k$ in $T=\left(\ldots, a_{i j}, \ldots\right)$ for each $x \in \mathbb{C}^{n+1}$ and for each index $I$. So the equation $\omega(x) \wedge\left(T^{*} \omega\right)(x)=0$ is equivalent to the vanishing of the polynomials $H_{I J}^{x}\left(\ldots, a_{i j}, \ldots\right)=A_{I}(x) B_{J}^{x}\left(\ldots, a_{i j}, \ldots\right)-A_{J}(x) B_{I}^{x}\left(\ldots, a_{i j}, \ldots\right)$ for all $I, J$ with $|I|=|J|=k$.

Therefore $\operatorname{Aut}(\mathcal{W})$ is an intersection of hypersurfaces $Z_{I J}^{x}=\left(H_{I J}^{x}=0\right)$ of degree $d+2 k$ in $\mathbb{P}^{r}$. By hypothesis, $\operatorname{Aut}(\mathcal{W})$ is finite. Then we can choose $r$ among the $Z_{I J}^{x}$, say $Z_{1}, \ldots, Z_{r}$ such that $Z_{i}$ does not contain any irreducible component of $Z_{1} \cap \cdots \cap Z_{i-1}$ for each $i=1, \ldots, r$, and $\operatorname{Aut}(\mathcal{W})=\cap_{i=1}^{r} Z_{i}$. It follows from Bézout's Theorem that

$$
|\operatorname{Aut}(\mathcal{W})| \leq(d+2 k)^{(N+1)^{2}-1}
$$

In particular, we obtain a polynomial upper bound for the order of the self-birational group of a generic foliation $\mathcal{F}$ on $\mathbb{P}^{2}$ depending only on its degree.

Corollary 3.2. If $\mathcal{F}$ is a reduced foliation on $\mathbb{P}^{2}$ of degree $d>1$, then $\operatorname{Bir}(\mathcal{F})=\operatorname{Aut}(\mathcal{F})$ and

$$
|\operatorname{Bir}(\mathcal{F})| \leq(d+2)^{8} .
$$

Proof. Since $K_{\mathcal{F}}=\mathcal{O}_{\mathbb{P}^{2}}(d-1)$ is ample, follows from [2, Theorem 1 p.75] that there exists a unique minimal model for $\left(\mathbb{P}^{2}, \mathcal{F}\right)$. The minimal model is obtained in two steps: first resolve the singularities of the foliation and then contract exceptional invariant curves. But the singularities are already reduced and $\mathbb{P}^{2}$ does not admit $(-1)$-curves. Therefore $\left(\mathbb{P}^{2}, \mathcal{F}\right)$ is minimal and consequently $\operatorname{Bir}(\mathcal{F})=\operatorname{Aut}(\mathcal{F})$. To conclude the proof we have just to apply Theorem 2.1 and Proposition 3.1 . 
3.3. Duality. Let $\check{\mathbb{P}}^{N}$ denote the projective space parametrizing hyperplanes in $\mathbb{P}^{N}$ and $\mathcal{I}$ be the incidence variety, that is

$$
\mathcal{I}=\left\{(x, H) \in \mathbb{P}^{N} \times \check{\mathbb{P}}^{N} ; x \in H\right\} .
$$

If $X \subset \mathbb{P}^{N}$ is a projective variety of dimension $n$, the conormal variety $\operatorname{Con}(\mathrm{X})$ is the closure in $\mathcal{I}$ of the set of pairs $(x, H)$ such that $x$ is a smooth point of $X$ and $H$ is a hyperplane containing the tangent plane $T_{x} X$.

Now let $\mathcal{W}$ be an irreducible codimension one $k$-web on $X$. The conormal variety of the pair $(X, \mathcal{W})$ is the subvariety $\operatorname{Con}(X, \mathcal{W})$ of $\mathcal{I}$ defined by

$$
\operatorname{Con}(X, \mathcal{W})=\overline{\left\{(x, H) \in \mathcal{I} ; x \in \mathcal{W}_{s m} \text { and } \exists i, 1 \leq i \leq k, H \supset T_{x}^{i} \mathcal{W}\right\}}
$$

where the overline in the right side means the Zariski closure in $\mathcal{I}$. We note that for $x \in \mathcal{W}_{s m}$ the fiber $\pi^{-1}(x) \cap \operatorname{Con}(X, \mathcal{W})$ is a union of $k$ linear spaces of dimension $N-n$, then $\operatorname{Con}(X, \mathcal{W})$ has codimension $N-1$ in $\mathcal{I}$.

Using the natural identification of $\mathcal{I}$ with $\mathbb{P}\left(T^{*} \mathbb{P}^{N}\right)$ the conormal $\operatorname{Con}(X, \mathcal{W})$ can be also characterizad by the following conditions (see [15, Section 1.4]):

(1) $\operatorname{Con}(X, \mathcal{W})$ is irreducible;

(2) $\pi(\operatorname{Con}(X, \mathcal{W}))=X$

(3) For generic $x \in X$ the fiber $\pi^{-1}(x) \cap \operatorname{Con}(X, \mathcal{W})$ is a union of linear subspaces corresponding to the projectivization of the conormal bundles in $\mathbb{P}^{N}$ of the leaves of $\mathcal{W}$ passing through $x$.

Given $x \in \mathcal{W}_{s m}$ we have the superposition of $k$ distinct codimension one foliations in a neighborhood $U$ of $x$ in $X$. The lift of these foliations to $\mathcal{I}$ determines a codimension one foliation $\mathcal{F}_{U}$ on $\pi^{-1}(U) \cap \operatorname{Con}(X, \mathcal{W})$ in which is just the foliation obtained by the restriction of the contact form $\alpha \in \mathrm{H}^{0}\left(\mathcal{I}, \Omega_{\mathcal{I}}^{1} \otimes \mathcal{O}_{\mathcal{I}}(1)\right)$ to $\pi^{-1}(U) \cap \operatorname{Con}(X, \mathcal{W})$ (see [15, p. 39] for details). So we get a codimension one foliation $\mathcal{F}$ globally defined on $\operatorname{Con}(X, \mathcal{W})$ such that the leaves are the lifting of leaves of $\mathcal{W}$.

In order to obtain the dual pair $\mathcal{D}(X, \mathcal{W})$ we consider the variety $Y=\check{\pi}(\operatorname{Con}(X, \mathcal{W})) \subset \check{\mathbb{P}}^{N}$. Let $s$ be the number of irreducible components of the fiber $\check{\pi}^{-1}(H) \cap \operatorname{Con}(X, \mathcal{W})$ for generic $H \in Y$. If $\operatorname{dim} Y>1$, over regular values of $\check{\pi}$ the direct image of $\mathcal{F}$ can be identified with the superposition of $s$ distinct codimension one foliations. Therefore we arrive in a codimension one web $\mathcal{W}^{\vee}$ on $Y$. If $\operatorname{dim} Y=1$ the leaves of $\mathcal{F}$ are projected in points. In this case we adopt the convention that on the irreducible curve $Y$ there is only one web $\mathcal{W}^{\vee}$, the 1-web which has as leaves the points of $Y$. We shall say that $\mathcal{D}(X, \mathcal{W})=\left(Y, \mathcal{W}^{\vee}\right)$ is the dual pair of $(X, \mathcal{W})$.

The characteristic numbers associated to the pair $(X, \mathcal{W})$ are

$$
d_{i}(X, \mathcal{W})=\int_{\mathcal{I}}[\operatorname{Con}(X, \mathcal{W})]\left[\operatorname{Con}\left(\mathbb{P}^{i}\right)\right]
$$

where $i=0, \ldots, N-1$ and $\mathbb{P}^{i} \subset \mathbb{P}^{N}$. Notice that if $X=\mathbb{P}^{N}$ then $d_{0}(X, \mathcal{W})=k$ and $d_{1}(X, \mathcal{W})=$ $\operatorname{deg}(\mathcal{W})$. The last equality follows from the fact that $\operatorname{Con}\left(\mathbb{P}^{1}\right)$ is the set of points $(x, H)$ such that $x \in \mathbb{P}^{1}$ and $H$ contains $\mathbb{P}^{1}$. So if $\mathbb{P}^{1}$ represents a generic line, $x \in \operatorname{Con}(X, \mathcal{W}) \cap \operatorname{Con}\left(\mathbb{P}^{1}\right)$ if and only if $x$ is contained in the tangency locus between $\mathcal{W}$ and $\mathbb{P}^{1}$. We also observe that since $\operatorname{Con}(X, \mathcal{W})=\operatorname{Con} \mathcal{D}(X, \mathcal{W})$ and $\operatorname{Con}\left(\mathbb{P}^{i}\right)=\operatorname{Con}(L)$ where $L \simeq \mathbb{P}^{N-i-1} \subset \check{\mathbb{P}}^{N}$ one obtains

$$
d_{i}(X, \mathcal{W})=d_{N-i-1} \mathcal{D}(X, \mathcal{W})
$$

\section{Main Results}

Given a smooth projective variety $X$ of dimension $n$ and a codimension one foliation $\mathcal{F}$ on $X$, with ample canonical bundle $K_{\mathcal{F}}$, the $m$-th pluricanonical map

$$
\phi_{m}: X \longrightarrow \mathbb{P}^{N}=\mathbb{P H}^{0}\left(X, K_{\mathcal{F}}^{\otimes m}\right)^{\vee}
$$

is an embedding for sufficiently large $m$. The map $\phi_{m}$ send a point $p \in X$ to the hyperplane in $\mathbb{P} H^{0}\left(X, K_{\mathcal{F}}^{\otimes m}\right)$ consisting of the sections vanishing at $p$. Let us denote by $X_{m}=\phi_{m}(X) \subset \mathbb{P}^{N}$ 
the embedded variety and $\mathcal{F}_{m}$ the respective foliation on $X_{m}$. Since $\operatorname{Aut}(\mathcal{F})$ acts naturally in $\mathbb{P H}^{0}\left(X, K_{\mathcal{F}}^{\otimes m}\right)$ and so in $\mathbb{P H}^{0}\left(X, K_{\mathcal{F}}^{\otimes m}\right)^{\vee}$, this action induces a monomorphism of groups

$$
\operatorname{Aut}(\mathcal{F}) \longrightarrow \operatorname{PGL}(N, \mathbb{C})
$$

which image in $\operatorname{PGL}(N, \mathbb{C})$ is exactly $\operatorname{Lin}\left(\mathcal{F}_{m}\right)$, the automorphisms of $\mathbb{P}^{N}$ leaving $X_{m}$ and $\mathcal{F}_{m}$ invariant.

The following remark will be useful in the sequel.

Remark 4.1. Matsusaka's Big Theorem states that given an ample line bundle $\mathcal{L}$ on a smooth projective variety $X$ of dimension $n$, there exists a positive integer $m_{0}$, depending only on the coefficients of the Hilbert polynomial of $\mathcal{L}$ such that $\mathcal{L}^{\otimes m}$ is very ample for $m \geq m_{0}$. This theorem was improved by J. Kollár and T. Matsusaka in 13 showing that $m_{0}$ depends only on the coefficients $t^{n}$ and $t^{n-1}$ in the Hilbert Polynomial $P(t)$. This implies that $m_{0}$ depends only on $\mathcal{L}^{n}$ and $\mathcal{L}^{n-1} K_{X}$. We will need of an effective version of Matsusaka's Big Theorem obtained by G. Fernandez del Busto in [8]. He proves that if $\mathcal{L}$ is an ample line bundle on a smooth projective surface $X$ and

$$
m>k_{0}=\frac{1}{2}\left[\frac{\left(\mathcal{L} \cdot\left(K_{X}+4 \mathcal{L}\right)+1\right)^{2}}{\mathcal{L}^{2}}+3\right]
$$

then $\mathcal{L}^{\otimes m}$ is very ample.

Now we are able to prove our main result. The idea of the proof is in some sense close to the argument of [10, Theorem 1]. That is, first apply Matsusaka's Big Theorem to embed $X$, goes to the dual space and then use homogeneous coordinates to bound the order of $\operatorname{Aut}(\mathcal{F})$. In our case, the dual of the pair $(X, \mathcal{F})$ is a codimension one web.

Theorem 4.2. Let $\mathcal{F}$ be a foliation on a smooth irreducible projective surface $X$. If $K_{\mathcal{F}}$ is ample and $\operatorname{Aut}(\mathcal{F})$ is finite then

$$
|\operatorname{Aut}(\mathcal{F})| \leq\left(\left(3 m^{2}+2 m\right) K_{\mathcal{F}}^{2}\right)^{\left(m^{2} K_{\mathcal{F}}^{2}+2\right)^{2}-1}
$$

where $m=\left(K_{\mathcal{F}} \cdot\left(K_{X}+4 K_{\mathcal{F}}\right)+1\right)^{2}+3 K_{\mathcal{F}}^{2}$.

Proof. Let us fix $m=\left(K_{\mathcal{F}} \cdot\left(K_{X}+4 K_{\mathcal{F}}\right)+1\right)^{2}+3 K_{\mathcal{F}}^{2}$. By Remark 4.1, $K_{\mathcal{F}}^{\otimes m}$ is very ample and hence the $m$-pluricanonical map is an embedding. Let $X_{m} \subset \mathbb{P}^{N}$ be the embedded variety and $\mathcal{F}_{m}$ the respective foliation on $X_{m}$. We will first prove that the dual web $\mathcal{W}_{m}=\mathcal{F}_{m}^{\vee}$ is a codimension one web on $\check{\mathbb{P}}^{N}$. Since the dual variety of a curve, in which is not a line, is always a hypersurface, we must prove that a generic leaf of $\mathcal{F}_{m}$ is not a line. If a leaf $L$ of $\mathcal{F}_{m}$ is a line then $L$ must pass through a singular point of the foliation. In fact, if $L$ does not contain a singular point then the restriction of $K_{\mathcal{F}_{m}}$ to this leaf coincides with the canonical bundle $K_{L}=\mathcal{O}_{\mathbb{P}^{1}}(-2)$ of $L$. This contradicts the hypothesis of $K_{\mathcal{F}_{m}}$ be ample. Therefore if a generic leaf of $\mathcal{F}_{m}$ is a line, there are an infinite quantity passing through the same singular point $x \in \operatorname{Sing}\left(\mathcal{F}_{m}\right)$. Since $X$ is smooth irreducible this is enough to ensure that $X$ is a linearly embedded $\mathbb{P}^{2}$ and $\mathcal{F}_{m}$ is the foliation by lines passing through $x$. The last assertion follows from the fact that the tangence between two foliations is an algebraic subset of $X$. But, in this case, $K_{\mathcal{F}_{m}}=\mathcal{O}_{\mathbb{P}^{2}}(-1)$ is not ample.

The image of $\operatorname{Aut}(\mathcal{F})$ in $\operatorname{PGL}(N, \mathbb{C})$ by the monomorphism

$$
\operatorname{Aut}(\mathcal{F}) \longrightarrow \operatorname{PGL}(N, \mathbb{C})
$$

is $\operatorname{Lin}\left(\mathcal{F}_{m}\right)$, the automorphisms of $\mathbb{P}^{N}$ leaving $X_{m}$ and $\mathcal{F}_{m}$ invariant. $\operatorname{Since} \operatorname{Lin}\left(\mathcal{F}_{m}\right) \simeq \operatorname{Aut}\left(\mathcal{W}_{m}\right)$ we have just to bound $\operatorname{Aut}\left(\mathcal{W}_{m}\right)$. By hypothesis $\operatorname{Aut}(\mathcal{F})$ is finite, and thus one obtains the same conclusion for $\operatorname{Aut}\left(\mathcal{W}_{m}\right)$. It follows from Proposition 3.1 that

$$
\left|\operatorname{Aut}\left(\mathcal{W}_{m}\right)\right| \leq(d+2 k)^{(N+1)^{2}-1} .
$$

But from (11),

$$
d=d_{1}\left(\check{\mathbb{P}}^{N}, \mathcal{W}_{m}\right)=d_{N-2}\left(X_{m}, \mathcal{F}_{m}\right)
$$

and

$$
k=d_{0}\left(\check{\mathbb{P}}^{N}, \mathcal{W}_{m}\right)=d_{N-1}\left(X_{m}, \mathcal{F}_{m}\right)
$$


Now we can write this numbers intrinsically. The second one is the number of tangencies between $\mathcal{F}_{m}$ and a generic hyperplane section of $X_{m}$. That is,

$$
d_{N-1}\left(X_{m}, \mathcal{F}_{m}\right)=\operatorname{tang}\left(\mathcal{F}_{m}, \mathcal{O}_{X_{m}}(1)\right)=\operatorname{tang}\left(\mathcal{F}, K_{\mathcal{F}}^{\otimes m}\right)
$$

Thus by [2, Proposition 2 p.23], $d_{N-1}\left(X_{m}, \mathcal{F}_{m}\right)=\left(m^{2}+m\right) K_{\mathcal{F}}^{2}$. It is not hard to see that the first number $d_{N-2}\left(X_{m}, \mathcal{F}_{m}\right)$ coincides with $\operatorname{deg}\left(X_{m}\right)$, hence $d_{N-2}\left(X_{m}, \mathcal{F}_{m}\right)=m^{2} K_{\mathcal{F}}^{2}$. Therefore

$$
\left|\operatorname{Aut}\left(\mathcal{W}_{m}\right)\right| \leq\left(\left(3 m^{2}+2 m\right) K_{\mathcal{F}}^{2}\right)^{(N+1)^{2}-1} \text {. }
$$

Since $N=h^{0}\left(X, K_{\mathcal{F}}^{\otimes m}\right)-1$, to conclude the proof of the theorem we need only to apply the following bound given in [14, Theorem 3]

$$
h^{0}\left(X, K_{\mathcal{F}}^{\otimes m}\right) \leq m^{2} K_{\mathcal{F}}^{2}+2 .
$$

Corollary 4.3. Let $\mathcal{F}$ be a foliation on a non-rational smooth irreducible projective surface $X$. If $K_{\mathcal{F}}$ is ample then

$$
|\operatorname{Aut}(\mathcal{F})| \leq\left(\left(3 m^{2}+2 m\right) K_{\mathcal{F}}^{2}\right)^{\left(m^{2} K_{\mathcal{F}}^{2}+2\right)^{2}-1}
$$

where $m=\left(K_{\mathcal{F}} \cdot\left(K_{X}+4 K_{\mathcal{F}}\right)+1\right)^{2}+3 K_{\mathcal{F}}^{2}$.

Proof. The finiteness of $\operatorname{Aut}(\mathcal{F})$ is ensured by Proposition 2.2

In the following corollary, the pair $(X, \mathcal{F})$ represents a foliation $\mathcal{F}$ on a smooth irreducible projective surface $X$.

Corollary 4.4. There exists a function $f$, so that if $(X, \mathcal{F})$ is reduced and has ample canonical bundle $K_{\mathcal{F}}$, then the minimal model $(Y, \mathcal{G})$ has ample canonical bundle $K_{\mathcal{G}}$ and

$$
|\operatorname{Bir}(\mathcal{F})| \leq f\left(K_{\mathcal{G}}^{2}, K_{\mathcal{G}} K_{Y}\right)
$$

Proof. We proceed similarly to the proof of Corollary 3.2. Let $(Y, \mathcal{G})$ the minimal model of $(X, \mathcal{F})$. The minimal model is obtained contracting exceptional invariant curves $\pi:(X, \mathcal{F}) \longrightarrow(Y, \mathcal{G})$ and the contraction of an exceptional curve produces a reduced singularity or a regular point. Thus $\pi$ is a sequence of blowing-ups over reduced singularities or regular points.

We will show that amplitude of $K_{\mathcal{F}}$ implies amplitude of $K_{\mathcal{G}}$. In general if $\pi:(X, \mathcal{F}) \longrightarrow$ $\left(Y_{1}, \mathcal{G}_{1}\right)$ is the blow up over a singularity $x$ of $\mathcal{G}_{1}$ and $l_{x}$ is the order of $\mathcal{G}_{1}$ on $E=\pi^{-1}(x)$ then $K_{\mathcal{F}}=\pi^{*}\left(K_{\mathcal{G}_{1}}\right) \otimes \mathcal{O}\left(\left(1-l_{x}\right) E\right)$, see [2, p. 26]. If $x$ is a reduced singularity then $K_{\mathcal{F}}=\pi^{*}\left(K_{\mathcal{G}_{1}}\right)$ and if $x$ is a regular point then $K_{\mathcal{F}}=\pi^{*}\left(K_{\mathcal{G}_{1}}\right) \otimes \mathcal{O}(E)$. Thus $K_{\mathcal{F}}$ ample implies that $K_{\mathcal{G}_{1}}$ is ample, as can be checked by using Nakai-Moishezon Criterion. This is enough to deduce the amplitude of $K_{\mathcal{G}}$.

To finish the proof of the corollary we observe that $\operatorname{Bir}(\mathcal{F})=\operatorname{Bir}(\mathcal{G})=\operatorname{Aut}(\mathcal{G})$ and apply Theorem 2.1 together with Theorem 4.2.

Remark 4.5. We remember that by definition, $\mathcal{F}$ is of general type if and only if $\mathcal{F}$ has a reduced model $\tilde{\mathcal{F}}$ with big canonical bundle $K_{\tilde{\mathcal{F}}}$. Corollary 4.4 can be applied to foliations $\mathcal{F}$ with the stronger hypothesis on $K_{\tilde{\mathcal{F}}}$ to be ample.

\section{REFERENCES}

[1] A. Andreotti, Sopra le superficie che possegono transformazioni birazionali in se, Red. Mat. e Appl. 9, 1950, 255-279.

[2] M. Brunella, Birational Geometry of Foliations, IMPA publications, (2000). (see http://www.impa.br/opencms/en/biblioteca).

[3] S. Boucksom, C. Favre and M. Jonsson, Degree growth of meromorphic surface maps, Duke Math. Journal 141 (2008), no.3, 519-538.

[4] S. Cantat and C. Favre, Symétries birationnelles des surfaces feuilletées, J. Reine Angew. Math. 561 (2003), 199-235. Corrigendum. J. Reine Angew. Math. 582 (2005), 229231.

[5] F. Catanese and M. Schneider, Polynomial bounds for abelian groups of automorphisms, Compositio Math. 97 (1995), no. 1-2, 115, Special issue in honour of Frans Oort.

[6] A. Corti, Polynomial bounds for the number of automorphisms of a surface of general type, Ann. Sci. École Norm. Sup. (4) 24 (1991), no. 1, 113137. 
[7] D. Eisenbud, Commutative Algebra with a View Towards Algebraic Geometry, GTM 150, Springer-Verlag (1995).

[8] G. Fernandez del Busto, A Matsusaka type theorem on surfaces. J. Algebraic Geom. 5 (1996), 513-520

[9] C. D. Hacon, J. McKernan and C. Xu, On the birational automorphisms of varieties of general type, arXiv:1011.1464 1 .

[10] A. Howard and A.J. Sommese, On the orders of the automorphism groups of certain projective manifolds, J. Hano et al. (Eds.): Manifolds and Lie Groups. Progress in Math. 14, Birkhauser, (1981), pp. 145-158.

[11] A. T. Huckleberry and M. Sauer, On the order of the automorphism group of a surface of general type, Math. Zeitschrift 205 (1990), no. 2, 321329.

[12] A. Hurwitz, ber algebraische Gebilde mit eindeutigen Transformationen in sich. Math. Ann. 41, 403-442, 1893.

[13] J. Kollár and T. Matsusaka, Riemann-Roch type inequalities. Amer. J. Math. 105 (1983), 229-252.

[14] T. Matsusaka and D. Mumford, Two fundamental theorems on deformations of polarized varieties, Amer. J. Math. 86 (1964), 668-684

[15] J.V. Pereira and L. Pirio, An invitation to web geometry. Publicações Matemáticas do IMPA, (2009).

[16] J.V. Pereira and P.F Sanchez, Transformation groups of holomorphic foliations. Commun. Anal. Geom. 10(5), 1115-1123 (2002)

[17] A. Seidenberg, Reduction of singularities of the differential equation Ady $=B d x$, Amer. J. Math. 89 (1968), 248-269.

[18] E. Szabó, Bounding automorphism groups, Math. Ann. 304 (1996), no. 4, 801811.

[19] H. Tsuji, Bound of automorphisms of projective varieties of general type, arXiv:math/0004138 1.

[20] G. Xiao, Bound of automorphisms of surfaces of general type. I, Ann. of Math. 2139 (1994), no. 1, 5177.

[21] G. Xiao, Bound of automorphisms of surfaces of general type. II, J. Algebraic Geom. 4 (1995), no. 4, 701793.

[22] G. Xiao, Linear bound for abelian automorphisms of varieties of general type, J. Reine Angew. Math. 476 (1996), 201207.

[23] De-Qi Zhang, Small bound for birational automorphism groups of algebraic varieties, Math. Ann. 339 (2007), no. 4, 957975, With an appendix by Yujiro Kawamata.

Universidade Federal de Viçosa, Departamento de Matemática, Avenida P.H. Rolfs, s/n, 36570-000 ViçOSA MG BRAsil

E-mail address: mauricio.correa@ufv.br

Universidade Federal Fluminense, Instituto de Matemática, Departamento de Análise., Rua Mário Santos Braga, S/N, Valonguinho, 24020-140 Niterói RJ Brasil

E-mail address: tfassarella@id.uff.br 